Volume 2, Issue 2, April-June 2017, Pages: 190, DOI: http://dx.doi.org/10.19082/ah190

\title{
GLANCING OF CANCER REGISTRY SURVEILLANCE SYSTEM IN IRAN
}

\author{
Seyed Hossein Hosseini*1, Dr. Seyed Mohammad Hashemi², Dr. Hasan Makarem ${ }^{3}$
}

1: Graduate Student of Epidemiology, School of Public Health, Shahid Beheshti University of Medical Sciences, Tehran, Iran.

2: General Medicine, Health Network Management Khalil Abad, Mashhad University of Medical Sciences, Iran.

3: General Medicine, Health Center director Khalil Abad, Mashhad University of Medical Sciences, Iran

Correspondence:

Tel:+985157727313, Fax:+985157727315, E-mail: hoseinih8@mums.ac.ir

TYPE OF ARTICLE: CONFERENCE ABSTRACT

\begin{abstract}
Introduction: Currently, more than 25 million people worldwide are living with cancer each year. Additionally, more than 11 million people are diagnosed and 7 million deaths from cancer will occur. It is anticipated that the above statistics for 2030 are respectively, 17, 27 and 75 million, and this growth will be mainly related to developing countries. After cardiovascular diseases and injuries, cancer is the third leading cause of death in Iran. Every year over 30,000 Iranians lose their lives from cancer,. It is estimated that each year, more than 80,000 new cases of cancer will occur in the country. With increasing life expectancy, an increase in the percentage of elderly in the population is expected to double current incidence of cancer in the next two decades.

Methods: The study was a descriptive study using different sources of studies on cancer registration in Iran.

Results: Following mandatory reporting of cancerous diseases approval of parliament in 1984, and with law enforcement in 1986, the first cancer registries based on pathology reports collected from pathology centers, both public and private, from across the country were carried out in 1986. Although pathological evidence-based cancer registry system has been able to cost slightly more than 80 percent of cancer cases in the country to identify and register. Although the cancer registry system in Iran is successful in the field of public health, for some cancers registered, the data are low in number. Cancers of the pancreas, liver and lungs can be named as cancers that usually have no sample diagnosis based on clinical evidence. It also limits the system can be cited to track cases Which in some cases do not keep track of the Cancer Treatment Centers and patients with increased tumor stage encounter.

Conclusion: Targeted cancer data and research to determine the prevalence of cancer interventions to reduce cancer in each region to identify patients in the early stages of the disease, using the experiences of developed countries in the cancer registry are required. Furthermore, interventions with the integrity of the registration system resources with other pathologies such as cancer, death certificates, civil registration health centers, etc., are also required.
\end{abstract}

KEYWORDS: surveillance system, registration, cancer, Iran.

\footnotetext{
Abstracts of First National Congress of Medical Informatics, Mashhad, Iran, February 2017

(C) 2017 The Authors. This is an open access article under the terms of the Creative Commons Attribution-NonCommercialNoDerivs License, which permits use and distribution in any medium, provided the original work is properly cited, the use is non-commercial and no modifications or adaptations are made.
} 